\title{
SMA observations of Infrared Dark Clouds: A tale of two cores
}

\author{
J. M. Rathborne \\ Harvard-Smithsonian Center for Astrophysics, Mail Stop 42, 60 Garden Street, Cambridge, \\ MA 02138,USA; jrathborne@cfa.harvard.edu \\ J. M. Jackson \\ Institute for Astrophysical Research, Boston University, Boston, MA 02215; \\ jackson@bu.edu \\ Q. Zhang \\ Harvard-Smithsonian Center for Astrophysics, Mail Stop 42, 60 Garden Street, Cambridge, \\ MA02138,USA; qzhang@cfa.harvard.edu \\ R. Simon \\ I.Physikalisches Institut, Universität zu Köln, 50937 Köln, Germany; \\ simonr@ph1.uni-koeln.de
}

\begin{abstract}
We present high-angular resolution sub-millimeter continuum images and molecular line spectra obtained with the Submillimeter Array toward two massive cores that lie within Infrared Dark Clouds; one actively star-forming (G034.43+00.24 MM1) and the other more quiescent (G028.53-00.25 MM1). The high-angular resolution sub-millimeter continuum image of G034.43+00.24 MM1 reveals a compact $(\sim 0.03 \mathrm{pc})$ and massive $(\sim$ $\left.29 \mathrm{M}_{\odot}\right)$ structure while the molecular line spectrum shows emission from numerous complex molecules. Such a rich molecular line spectrum from a compact region indicates that G034.43+00.24 MM1 contains a hot molecular core, an early stage in the formation of a high-mass protostar. Moreover, the velocity structure of its ${ }^{13} \mathrm{CO}(3-2)$ emission indicates that this $\mathrm{B} 0$ protostar may be surrounded by a rotating circumstellar envelope. In contrast, the sub-millimeter continuum image of G028.53-00.25 MM1 reveals three compact $(\lesssim 0.06 \mathrm{pc})$, massive $(9-$ $\left.21 \mathrm{M}_{\odot}\right)$ condensations but with no lines detected in its spectrum. We suggest that the core G028.53-00.25 MM1 is in a very early stage in the high-mass starformation process; its size and mass are sufficient to form at least one high-mass star, yet it shows no signs of localized heating. Because the combination of high
\end{abstract}


velocity line wings with a large IR-mm bolometric luminosity $\left(\sim 10^{2} \mathrm{~L}_{\odot}\right)$ indicates that this core has already begun to form accreting protostars, we speculate that the condensations may be in the early phase of accretion and may eventually become high-mass protostars. We, therefore, have found the possible existence of two high-mass star-forming cores; one in a very early phase of star-formation and one in the later hot core phase. Together the properties of these two cores support the idea that the earliest stages of high-mass star-formation occur within IRDCs.

Subject headings: dust, extinction-stars:formation-ISM:clouds-infrared:ISM-radio lines:ISM

\section{Introduction}

Emerging evidence suggests that the very earliest stages of high-mass star and cluster formation occur within cold, dense molecular clumps called infrared dark clouds (IRDCs; Simon et al. 2006b; Rathborne et al. 2006, 2007). Because these molecular clumps have very high column densities $\left(\sim 10^{23}-10^{25} \mathrm{~cm}^{-2}\right)$ and low temperatures $(<25 \mathrm{~K})$, they have predominantly been identified via their absorption of the background Galactic emission at mid-IR wavelengths (e.g., Egan et al. 1998; Carey et al. 1998, 2000; Simon et al. 2006a). Because IRDCs are the densest clumps in molecular clouds (Simon et al. 2006b) which are now undergoing the process of fragmentation and condensation, IRDCs are important laboratories to study the pristine, undisturbed physical conditions of cluster-forming clouds before they are shredded apart by stellar winds and radiation.

Millimeter/sub-millimeter continuum studies of IRDCs Lis \& Carlstrom 1994; Carev et al. 2000; Garay et al. 2004; Ormel et al. 2005; Beuther et al. 2005; Rathborne et al. 2005, 2006) reveal that IRDCs contain many compact cores. These cores have typical sizes of $<0.5 \mathrm{pc}$ and masses of $\sim 120 \mathrm{M}_{\odot}$ (Rathborne et al. 2006). While most of these cores contain little evidence for active star formation, some are associated with bright $24 \mu \mathrm{m}$ emission, broad molecular line emission, shocked gas, and maser emission, indicating that they are actively forming stars (e.g., Rathborne et al. 2005; Wang et al. 2006; Chambers et al. 2008). Indeed, a number of low- and intermediate-mass protostars (Carev et al. 2000; Redman et al. 2003) in addition to high-mass protostars (Beuther et al. 2005; Rathborne et al. 2005; Pillai et al. 2006; Wang et al. 2006) have been identified within IRDCs.

Hot Molecular Cores (HMCs) are associated with the early stages of high-mass star formation. They correspond to the stage immediately after a cold dense starless core has 
formed. During the later stages in their evolution, HMCs are often also associated with methanol masers and ultra-compact H II regions. Numerous examples of HMCs have been found throughout the Galaxy (e.g., Garav \& Lizano 1999; Kurtz et al. 2000; Churchwell 2002), including one within an IRDC (Rathborne et al. 2007). HMCs are compact $(<0.1$ pc), dense $\left(\sim 10^{5}-10^{8} \mathrm{~cm}^{-3}\right)$, and massive $\left(\sim 10^{2} \mathrm{M}_{\odot}\right.$ : Garay \& Lizano 1999; Kurtz et al. 2000; Churchwell 2002). Due to the internal heating from the central high-mass protostar $(\mathrm{T} \sim 100 \mathrm{~K})$, they are typically very luminous $\left(>10^{3} \mathrm{~L}_{\odot}\right)$ and show strong emission from complex molecules (e.g., Kurtz et al. 2000). Accretion disks are also inferred in the later stage of the HMC phase directly (Zhang 2005; Cesaroni et al. 2007) or indirectly (Kurtz et al. 2000; Beuther et al. 2002; Zhang et al. 2001, 2005) by the presence of molecular outflows and maser emission. The existence of HMCs in IRDCs establishes a possible link between IRDCs and the early stages of high-mass star formation (Rathborne et al. 2007).

To understand the earliest stages in high-mass star formation one needs to identify and study the so-called 'high-mass starless cores,' the immediate precursors to high-mass protostars, HMCs, and ultra-compact $\mathrm{H}_{\text {II }}$ regions. High-mass star formation is rare and occurs rapidly; thus, the identification of high-mass starless cores is difficult. Because IRDCs have sizes, masses, and densities similar to cluster-forming molecular clumps but are considerably colder, we suggest that they are the precursors to clusters and their dense, cold cores the precursors to stars. Thus, the high-mass cores within IRDCs that contain no evidence for star formation are good candidates for the elusive 'high-mass starless cores.'

Here we present interferometric sub-millimeter continuum and molecular line observations obtained with the Submillimeter Array toward two cores within IRDCs; one showing evidence for active high-mass star formation (G034.43+00.24 MM1), the other showing none (G028.53-00.25 MM1). Both cores are at distances $\mathrm{d}>3.7 \mathrm{kpc}$ and have sizes $\mathrm{R}<0.8 \mathrm{pc}$, gas masses $\mathrm{M}>400 \mathrm{M}_{\odot}$, and bolometric luminosities $\mathrm{L}>10^{2} \mathrm{~L}_{\odot}$ (Table1, Simon et al. 2006b; Rathborne et al. 2006, 2008). We speculate that the 'active' core is in a later evolutionary stage than the more quiescent core. The new interferometric data reveal distinct properties for these cores and suggest that G034.43+00.24 MM1 is a more evolved high-mass protostar in the HMC phase and that G028.53-00.25 MM1 may be in a very early 'starless' core phase of high-mass star-formation. 


\section{Observations and Data Reduction}

\subsection{Interferometric continuum and spectral line data}

Interferometric observations of G034.43+00.24 MM1 and G028.53-00.25 MM1 were carried out with the Submillimeter Array 1 (SMA; Ho et al. 2004) on 2006 September 11 with seven antennas in the compact configuration. The projected baselines of the visibility data range from $15 \mathrm{~m}$ to $70 \mathrm{~m}$. The double-sideband receivers cover a total of $4 \mathrm{GHz}$ bandwidth. The digital correlator was configured to cover 329.1 to $331.1 \mathrm{GHz}$ for the lower sideband and 339.1 to $341.1 \mathrm{GHz}$ for the upper sideband with a uniform channel spacing of $0.8125 \mathrm{MHz}$ $\left(\sim 0.7 \mathrm{~km} \mathrm{~s}^{-1}\right)$ across the entire band. This setup resulted in simultaneous observations of the ${ }^{13} \mathrm{CO}(3-2), \mathrm{C}^{18} \mathrm{O}(3-2)$, $\mathrm{SO}\left(7_{8}-6_{7}\right)$, and ${ }^{34} \mathrm{SO}\left(8_{9}-7_{8}\right)$ transitions.

The $336 \mathrm{GHz}$ zenith opacities, measured with the NRAO tipping radiometer located at the Caltech Submillimeter Observatory, were $\tau \sim 0.4$ (scaled from the $225 \mathrm{GHz}$ measurement via $\left.\tau_{336 G H z}=2.8 \times \tau_{225 G H z}\right)$. The measured double-sideband system temperatures corrected to the top of the atmosphere were between 200 and $800 \mathrm{~K}$.

The primary beam of the SMA at these frequencies is $\sim 37^{\prime \prime}$. The phase centers for the observations are listed in Table 1. We used the quasars 1751+096 and 1743-038 to calibrate time dependent gains, and 3C454.3 to remove the gain variations across the passband. The flux scale was referenced to Uranus. The visibility data were calibrated with the IDL superset MIR package developed for the Owens Valley Radio Observatory (OVRO) Interferometer. The absolute flux level is accurate to about $25 \%$. After the calibration in MIR, the visibility data were exported to the MIRIAD format for further processing and imaging. The continuum is constructed from the line free channels in the visibility domain, and is further self-calibrated using the clean components of the image as input models. The gain solutions from the continuum self calibration is then applied to the spectral line data. The rms noise in the naturally weighted maps is $\sim 12$ and $\sim 4$ mJy beam $^{-1}$ in the continuum (for G034.43+00.24 MM1 and G028.53-00.25 MM1 respectively). The noise in the continuum map for G034.43+00.24 MM1 is higher because fewer line-free channels were available. For both cores the rms noise is $120 \mathrm{mJy}^{\text {beam }}{ }^{-1}(\sim 2.9 \mathrm{~K})$ per $1.0 \mathrm{~km} \mathrm{~s}^{-1}$ channel in the line data. The final synthesized beam was $\sim 2.3^{\prime \prime} \times 2.1^{\prime \prime}$ which corresponds to a physical size of $\sim 0.04 \mathrm{pc}(\sim 8,000 \mathrm{AU})$ and $\sim 0.06 \mathrm{pc}(\sim 12,600 \mathrm{AU})$ for G034.43+00.24 MM1 and G028.53-00.25 MM1 respectively.

\footnotetext{
${ }^{1}$ The Submillimeter Array is a joint project between the Smithsonian Astrophysical Observatory and the Academia Sinica Institute of Astronomy and Astrophysics, and is funded by the Smithsonian Institution and the Academia Sinica.
} 


\section{2. $\quad$ Single dish spectra}

To help with the interpretation of the SMA data, we have also included here lowerangular resolution spectra. These data were obtained as part of a large molecular line survey of cores within IRDCs conducted using the $30 \mathrm{~m}$ Institute de Radioastronomie Millimetrique (IRAM) telescope and the $15 \mathrm{~m}$ James Clerk Maxwell Telescope (JCMT). The molecular lines of $\mathrm{CS}(3-2), \mathrm{N}_{2} \mathrm{H}^{+}(1-0)$, $\mathrm{SiO}(2-1), \mathrm{H}^{13} \mathrm{CO}^{+}(1-0)$, and $\mathrm{HCO}^{+}(1-0)$ were obtained with IRAM in 2004 November, while the $\mathrm{HCN}(4-3),{ }^{13} \mathrm{CO}(3-2)$, and $\mathrm{C}^{18} \mathrm{O}(3-2)$ spectra were obtained with the JCMT in 2004 April. The IRAM spectra have a typical beam size of $\sim 26^{\prime \prime}$, an rms noise of $0.1 \mathrm{~K} \mathrm{channel}^{-1}$, and a velocity resolution of $0.07 \mathrm{~km} \mathrm{~s}^{-1}$. The JCMT spectra have a typical beam size of $\sim 14^{\prime \prime}$, an rms noise of $0.1 \mathrm{~K}_{\text {channel }}{ }^{-1}$, and a velocity resolution of $1.1 \mathrm{~km} \mathrm{~s}^{-1}$.

In both cases, chopper wheel calibration, pointing, and focus checks were performed regularly. All spectra were obtained in position-switched mode with a suitable nearby emissionfree position used as a reference (typically $\sim 5^{\prime}$ from the core). The data were reduced using standard methods in the software packages GILDAS and SPECX.

\section{Results}

\subsection{Low-angular resolution molecular line emission}

Figures 1 and 2 show the Spitzer $24 \mu \mathrm{m}$ images of the two IRDCs overlaid with the lowangular resolution (11") IRAM $30 \mathrm{~m} 1.2 \mathrm{~mm}$ continuum emission (Rathborne et al. 2006). Note that while both IRDCs remain dark at $24 \mu \mathrm{m}$, the core G034.43+00.24 MM1 shows bright emission at $24 \mu \mathrm{m}$.

Also included in these figures are the single dish low-angular resolution molecular line spectra obtained toward the cores. In both cases we see broad, saturated, and selfabsorbed lines. In particular, toward G034.43+00.24 MM1 we see broad line widths in the high-density tracers, CS (3-2) and HCN (4-3), shocked gas as traced by the bright $\mathrm{SiO}(2-1)$ emission, and high velocity line wings in $\mathrm{HCO}^{+}(1-0)$ and $\mathrm{H}^{13} \mathrm{CO}^{+}(1-0)$. Toward G028.53-00.25 MM1 we see saturated ${ }^{13} \mathrm{CO}(3-2)$ and $\mathrm{N}_{2} \mathrm{H}^{+}$(1-0) line emission, faint emission from $\mathrm{CS}(3-2)$ and $\mathrm{HCN}(4-3)$ with broad line wings in the $\mathrm{SiO}(2-1), \mathrm{HCO}^{+}(1-0)$, and $\mathrm{H}^{13} \mathrm{CO}^{+}(1-0)$ spectra.

Table 2 gives a summary of Gaussian fits to each of these spectra: peak $\mathrm{T}_{A}^{*}$, central $\mathrm{V}_{L S R}$, and FWHM line width, $\Delta V$. Because many of the lines show non-Gaussian profiles, the listed integrated intensity $(I)$ was calculated by summing the emission over a suitable 
velocity range $\left(\Delta V+5 \mathrm{~km} \mathrm{~s}^{-1}\right)$, rather than simply calculating the area under the Gaussian. For the $\mathrm{N}_{2} \mathrm{H}^{+}$(1-0) spectra, however, we do not fit a single Gaussian profile, but instead fit the hyperfine components simultaneously. The derived opacity for the main hyperfine components are 0.10 for G034.43+00.24 MM1 and 0.15 for G028.53-00.25 MM1. In these cases, the integrated intensity was calculated over the velocity range to include the emission from all hyperfine components.

\subsection{High-angular resolution continuum images}

Despite the similar sizes, masses, and bolometric luminosities (see Table 1) derived for these cores from the low-angular resolution continuum images, the high-angular resolution sub-millimeter continuum images show significant differences (Fig. 31). Toward G034.43+00.24 MM1 the emission remains unresolved, while toward G028.53-00.25 MM1 the emission is resolved into three individual condensations. In both cases the emission is coincident with the peak in the lower-angular resolution millimeter continuum images (shown as contours on Figs. 1 and (2). Table 3 lists the positions, deconvolved sizes, and peak fluxes which were determined from Gaussian fits to the emission within the high-angular resolution images.

\subsection{High-angular resolution molecular line emission}

The high-angular resolution molecular line data toward these two cores are also very different. Toward G034.43+00.24 MM1 the spectrum contains many molecular line emission features, but, in contrast, the spectrum toward G028.53-00.25 MM1 contains no significant molecular line emission whatsoever.

Figures 4 and 5 display the average spectra within $a \sim 2^{\prime \prime}$ box centered on the peak in the high-angular resolution continuum image toward G034.43+00.24 MM1 and G028.53-00.25 MM1 respectively. Toward G034.43+00.24 MM1, the spectra show strong emission from the ${ }^{13} \mathrm{CO}(3-2), \mathrm{C}^{18} \mathrm{O}(3-2)$, $\mathrm{SO}\left(7_{8}-6_{7}\right)$, and ${ }^{34} \mathrm{SO}\left(8_{9}-7_{8}\right)$ transitions in addition to numerous

\footnotetext{
${ }^{2}$ In this paper we use the term 'clump' to refer to IRDCs. These large $(\sim 5 \mathrm{pc})$, massive $\left(\sim 10^{3} \mathrm{M}_{\odot}\right)$, dense $\left(\sim 10^{3} \mathrm{~cm}^{-3}\right)$ molecular structures are found within a Giant Molecular Cloud (GMC) and will likely give rise to a cluster of stars. Within these 'clumps', the many compact substructures are referred to as 'cores' and have typical sizes of $\sim 0.5 \mathrm{pc}$ and masses of $\sim 10^{2} \mathrm{M}_{\odot}$. At high-angular resolution, these 'cores' can often be further resolved into multiple substructures. We refer to the small continuum substructures identified by the SMA, with sizes $\sim 0.05 \mathrm{pc}$, as 'condensations'. The 'condensations' presumably trace the material that will give rise to the individual stars.
} 
lines from the complex molecules $\mathrm{NH}_{2} \mathrm{CHO}, \mathrm{CH}_{2} \mathrm{NH}, \mathrm{CH}_{3} \mathrm{OCH}_{3}, \mathrm{CH}_{3} \mathrm{CN}$, and $\mathrm{CH}_{3} \mathrm{CH}_{2} \mathrm{CN}$. Such complex molecules are typically produced and excited in the immediate surroundings of a recently formed high-mass protostar as the grain ice mantles are evaporated (see Kurtz et al. 2000 and references therein). Moreover, the presence of these species in such a compact region $(\lesssim 0.03 \mathrm{pc})$ indicates that this is a HMC and, thus, an early stage in the formation of a high-mass protostar.

\section{Discussion}

\subsection{Gas masses, Jeans masses, Virial masses, and Jeans lengths}

The optically thin sub-millimeter continuum emission can be used to estimate the gas masses $\left(\mathrm{M}_{G}\right)$ of the condensations via the expression (Hildebrand 1983)

$$
M_{G}=\frac{F_{\nu} D^{2}}{\kappa_{\nu} B_{\nu}\left(T_{D}\right)}
$$

where $F_{\nu}$ is the observed integrated source flux density, $D$ is the distance, $\kappa_{\nu}$ is the dust opacity per gram of dust, and $B_{\nu}\left(T_{D}\right)$ is the Planck function at the dust temperature $\left(\mathrm{T}_{D}\right)$.

We estimate $\kappa_{0.87 \mathrm{~mm}}$ by scaling the value of $\kappa_{1.3 \mathrm{~mm}}\left(1.0 \mathrm{~cm}^{2} \mathrm{~g}^{-1}\right.$; Ossenkopf \& Henning 1994) by $\nu^{\beta}$, where $\beta$, the dust emissivity index. We assume a gas-to-dust mass ratio of 100 . In both cases we assume gray-body emission but with different dust temperatures and emissivity indices. Fits to the IR-millimeter spectral energy distributions (SEDs; Rathborne et al. 2008) for each core reveal dust temperatures of $34 \mathrm{~K}$ and $16 \mathrm{~K}$ and dust emissivity indices of 1.5 and 1.2 for G034.43+00.24 MM1 and G028.53-00.25 MM1 respectively (see Table 1). We assume these values for $\beta$ in the calculation of the condensation masses. For G034.43+00.24 MM1 we assume a $\mathrm{T}_{D}$ of $100 \mathrm{~K}$ because this is a typical temperature for HMCs (Kurtz et al. 2000).

An assumption of the dust temperature for the condensations within G028.53-00.25 MM1 is more difficult. Although fits to the core SED reveal a $\mathrm{T}_{D}$ of $16 \mathrm{~K}$, it is possible that the condensations may be small volumes of slightly warmer gas within this extended, cold envelope. Because strong emission at sub-millimeter wavelengths can trace either temperature or density enhancements ideally one needs high-angular resolution molecular line observations to directly measure the temperatures (e.g., $\mathrm{NH}_{3}$ ). The non-detection of any molecular lines in the high-angular resolution spectrum suggests that the temperature of the gas is quite cold. Thus, to calculate the masses for the condensations within G028.53-00.25 MM1 we assume a temperature of $16 \mathrm{~K}$. Because the temperature of the condensations may be higher than the temperature of the bulk of the gas within the core, the derived condensation masses 
may, therefore, be an upper limit to the true mass. For example, if the condensations have a temperature of $30 \mathrm{~K}$ rather than $16 \mathrm{~K}$, the derived masses will decrease by a factor of $\sim 2$.

At high-angular resolution, the derived mass for G034.43+00.24 MM1 is $29 \mathrm{M}_{\odot}$, while for G028.53-00.25 MM1 the condensation masses are 9-21 $\mathrm{M}_{\odot}$ (Table 3). The lower-angular resolution single dish millimeter continuum images reveal these cores to have masses of 430 and $1,130 \mathrm{M}_{\odot}$ respectively 3 . Thus, we find that only a small fraction $(\sim 4 \%)$ of the mass detected in the lower-angular resolution millimeter continuum data is recovered by the interferometer. Thus, it is likely that the emission traced by the interferometer corresponds to well-defined small, dense volumes of gas within a larger, more diffuse core.

To determine the likelihood that the cores and condensations will fragment further, we calculate both their Jeans $\left(\mathrm{M}_{J}\right)$ and Virial $\left(\mathrm{M}_{V}\right)$ masses. These masses were calculated using the expressions

$$
\begin{gathered}
M_{J} \approx 1.1 M_{\odot}\left[\frac{T_{D}}{10 \mathrm{~K}}\right]^{3 / 2}\left[\frac{\rho}{10^{-19} \mathrm{~g} \mathrm{~cm}^{-3}}\right]^{-1 / 2} \\
M_{\mathrm{V}}=1.145 \times 10^{3} \Delta V^{2} D \sqrt{\Omega} \quad M_{\odot}
\end{gathered}
$$

where $T_{D}$ is the dust temperature, $\rho$ is the mass density (Zinnecker \& Yorke 2007), $\Delta \mathrm{V}$ is the measured line width $\left(\mathrm{km} \mathrm{s}^{-1}\right), D$ is the distance $(\mathrm{kpc})$, and $\Omega$ is the area on the sky $\left(\operatorname{deg}^{2}\right)$. The mass density was estimated using the dust masses and by assuming the volume is equivalent to a sphere of radius $R$.

The Jeans masses for the cores and condensations were calculated assuming the dust temperatures listed in Tables 1 and 3 and assuming that the gas and dust temperatures are identical. One must exercise caution, however, in applying a temperature of $34 \mathrm{~K}$ for the core G034.43+00.24 MM1 as it is associated with a luminous $24 \mu \mathrm{m}$ source which may be heating the gas to well above $34 \mathrm{~K}$. For a gas temperature of $100 \mathrm{~K}, \mathrm{M}_{J}$ will be higher by a factor of $\sim 10$. While the HMC's temperature may be closer to $100 \mathrm{~K}$ and correspond to a small volume in the core, the bulk of gas is likely to be colder. If the actual gas temperature is higher by a factor of two than the assumed value, then $\mathrm{M}_{J}$ will increase by a factor of $\sim 4$.

In the case where thermal processes dominate, the ratio of the gas mass to Jeans mass $\left(\mathrm{M}_{G} / \mathrm{M}_{J}\right)$ estimates the degree to which a gas structure is likely to gravitationally collapse

\footnotetext{
${ }^{3}$ These masses differ slightly from those quoted in Rathborne et al. (2006) because of their assumption of a single dust temperature $(15 \mathrm{~K})$ and emissivity index (2.0) for all their cores.
} 
into thermally fragmented substructures. In contrast, if turbulence dominates, then the Jeans mass is no longer applicable and one must consider the Virial mass. In this case, the ratio of the gas mass to Virial mass $\left(\mathrm{M}_{G} / \mathrm{M}_{V}\right)$ is a better indicator of further fragmentation. In either case, however, a ratio greater than unity indicates that the gas is unstable and will further fragment.

Because the core line profiles in Figures 1 and 2 clearly show that the lines are much broader than what is expected from thermal broadening alone, it is likely that turbulence may significantly contribute to the pressure support within both cores. While we list the ratio $\mathrm{M}_{G} / \mathrm{M}_{J}$ for the cores and condensations in Tables 1 and 3 it is unlikely that this ratio is physically significant. Instead, we use the ratio $\mathrm{M}_{G} / \mathrm{M}_{V}$ to estimate the degree to which a gas structure is likely to gravitationally collapse into fragmented substructures.

To calculate $\mathrm{M}_{V}$ for the cores we use the line widths measured from the low-angular resolution optically thin $\mathrm{C}^{18} \mathrm{O}(3-2)$ emission (see Table2). For the condensations, we assume the same line width as measured toward the larger core. Because high-angular resolution $\mathrm{NH}_{3}$ observations toward a nearby IRDC show typical line widths of $\sim 1.2 \mathrm{~km} \mathrm{~s}^{-1}$ (Wang et al. 2006) on scales of the condensations, using the line width from the larger core may result in an overestimation of the calculated $\mathrm{M}_{V}$ for the condensations.

We find that $\mathrm{M}_{G} / \mathrm{M}_{V}$ is 2.8 and 2.3 for the cores G034.43+00.24 MM1 and G028.53-00.25 MM1 respectively (Table 11). For the condensations, we find that $\mathrm{M}_{G} / \mathrm{M}_{V}$ is $\lesssim 1$ (Table 3). Since $\mathrm{M}_{G} / \mathrm{M}_{V}$ is greater than unity for the cores, they are likely to fragment, but since $\mathrm{M}_{G} / \mathrm{M}_{V}$ is less than unity for the individual condensations, they are stable against further fragmentation and thus will probably give rise to individual stars.

The Jeans length for the cores and condensations was calculated using the expression

$$
\lambda_{J}=\sqrt{\frac{\pi k T_{D}}{G m_{p} \rho}}
$$

where $k$ is the Boltzmann constant, $T_{D}$ is the dust temperature, $G$ is the gravitational constant, $m_{p}$ is the mass of a proton, and $\rho$ is the mass density.

We find that the Jeans length, defined as the minimum length scale (radius) for gravitational fragmentation to occur, is smaller $(\sim 0.04 \mathrm{pc})$ for G034.43+00.24 MM1 than that measured toward G028.53-00.25 MM1 ( $\sim 0.16 \mathrm{pc}$; Table 1). The separation of the condensations within G028.53-00.25 MM1 ( $\sim 0.2 \mathrm{pc})$ is comparable to the Jeans length of this core. Moreover, on the smallest scales of the individual condensations, the Jeans lengths are comparable to the measured radii of the condensations (Table 3). These results support the idea that gravitational collapse is occurring within the IRDCs and that the fragmentation process has stopped at the smallest protostellar scales. 


\subsection{G034.43+00.24 MM1: a high-mass protostar with a rotating envelope?}

The core G034.43+00.24 MM1 shows many indicators of high-mass star formation, including a bright $24 \mu \mathrm{m}$ point source, broad molecular line emission, shocked gas, water maser emission, and molecular outflows (Garay et al. 2004; Shepherd et al. 2004; Rathborne et al. 2005, 2006; Wang et al. 2006). G034.43+00.24 MM1 also shows weak radio continuum emission consistent with a deeply embedded B2 protostar (Shepherd et al. 2004). Fits to its IR-millimeter SED reveal it contains an object with a bolometric luminosity of $\sim 10^{4.3} \mathrm{~L}_{\odot}$, consistent with a B0 protostar (Rathborne et al. 2008). Because its high-angular resolution sub-millimeter continuum flux is dominated by a single, compact, massive emission feature, it is likely that the luminosity is dominated by a single high-mass protostar.

Additional clues to the nature of this high-mass star-forming HMC can be obtained by examining the morphologies of its molecular line emission. For example, Figure 6 shows a distinct morphological difference between the $\mathrm{CH}_{3} \mathrm{CH}_{2} \mathrm{CN}$ and ${ }^{13} \mathrm{CO}$ emission. The 'hot core lines', in this case traced by the $\mathrm{CH}_{3} \mathrm{CH}_{2} \mathrm{CN}$ emission, are all unresolved at $\sim 0.03 \mathrm{pc}$ resolution, while the ${ }^{13} \mathrm{CO}$ emission is easily resolved into $\mathrm{a} \sim 0.12 \mathrm{pc}$ diameter extended region. Because the hot cores lines are excited in the immediate vicinity of a high-mass protostar, it is likely, therefore, that the unresolved $\mathrm{CH}_{3} \mathrm{CH}_{2} \mathrm{CN}$ emission is tracing the heated, compact $(\lesssim 0.03 \mathrm{pc}, \lesssim 6,500 \mathrm{AU})$ regions immediately surrounding the central protostar. On the other hand, the extended ${ }^{13} \mathrm{CO}$ emission is tracing the larger, less dense circumstellar region of the core.

This extended ${ }^{13} \mathrm{CO}$ circumstellar emission also reveals a clear velocity gradient with red-shifted emission to the southeast and blue-shifted emission to the northwest of the central protostar (Fig. (6)). Such a velocity gradient might arise from either an outflow or a rotating envelope. Observational evidence for accretion disks around high-mass protostars exists for only a few objects (see Cesaroni et al. 2007 and references therein). The widespread existence of large ( $>10,000 \mathrm{AU}$ ) disk-like structures in young HMCs would support the idea that high-mass stars form via monolithic collapse (e.g., McKee \& Tan 2003) rather than via competitive accretion (e.g. Bonnell \& Bate 2002). Because HMCs in IRDCs are probably very young, they are excellent candidates to search for high-mass accretion disks given their short lifetimes.

In recent OVRO observations of CO (1-0) emission, Shepherd et al. (2007) identify two outflows that may originate within the core G034.43+00.24 MM1. The dominant red-shifted lobe of the main outflow (their G34.4:A) appears to extend to the southwest, with little blue-shifted emission found to the northeast. Moreover, high-angular resolution $\mathrm{SiO}(1-0)$ imaging of this core with the VLA (Q. Zhang priv. comm.) confirms the presence and orientation of the $\mathrm{CO}$ outflow. This outflow appears to be orientated perpendicular to the 
extended ${ }^{13} \mathrm{CO}(3-2)$ structure found here. Thus, the orientation of the outflow is consistent with this extended ${ }^{13} \mathrm{CO}(3-2)$ structure being a rotating envelope surrounding the central high-mass protostar.

The position-velocity diagram of the circumstellar ${ }^{13} \mathrm{CO}(3-2)$ emission toward G034.43+00.24 MM1 is consistent with Keplerian rotation around a $M \sin (i) \sim 10-50 \mathrm{M}_{\odot}$ protostar (Fig. [7). Thus, we see tentative evidence for a rotating circumstellar envelope surrounding this protostar. The derived mass for the central protostar is consistent with the luminosity and spectral type determined from the core's SED $\left(10^{4.3} \mathrm{~L}_{\odot} ; \mathrm{B} 0\right)$. Higher angular and spectral resolution molecular line observations are required to better resolve the velocity field in order to confirm or refute the presence of an accretion disk.

\subsection{G028.53-00.25 MM1: an early phase of high-mass star-formation?}

In contrast to the bright, rich molecular line spectrum toward G034.43+00.24 MM1, the lack of strong molecular line emission toward G028.53-00.25 MM1 suggests that it is either colder, more extended, or both. While ${ }^{13} \mathrm{CO}(3-2)$ and $\mathrm{C}^{18} \mathrm{O}(3-2)$ emission is clearly seen toward this core in the lower-angular resolution data (Fig. 2, 14" beam), very little is seen in the high-angular resolution spectrum (Fig. 5. upper limit of $\sim 2.9 \mathrm{~K}$ ). Moreover, in the 14" single dish spectra, G028.53-00.25 MM1 shows only faint HCN (4-3) emission but does show self-absorbed CS (3-2), saturated or self-absorbed $\mathrm{N}_{2} \mathrm{H}^{+}(1-0)$, broad $\mathrm{SiO}(2-1)$ and $\mathrm{H}^{13} \mathrm{CO}^{+}(1-0)$, and an asymmetry in the $\mathrm{HCO}^{+}(1-0)$ emission. The lack of emission from the high excitation $\mathrm{HCN}$ transition and the saturated $\mathrm{N}_{2} \mathrm{H}^{+}$profile suggests that the molecular gas is cold with a very high optical depth. Because SiO emission typically traces outflow activity caused by shocked gas, it is usually detected only in star-forming cores. Thus, the detection of broad line wings in the $\mathrm{SiO}, \mathrm{HCO}^{+}$and $\mathrm{H}^{13} \mathrm{CO}^{+}$spectra suggests that this core may already harbor some star formation activity.

The absence of strong ${ }^{13} \mathrm{CO}(3-2)$ or $\mathrm{C}^{18} \mathrm{O}(3-2)$ emission in the high-angular resolution spectrum may imply that the emission seen in the lower-angular resolution spectrum arises from a cold extended envelope which is resolved out by the interferometer. Using the limit of $\sim 2.9 \mathrm{~K}$ for the brightness sensitivity of the SMA spectrum, we estimate from the single dish spectra that the sizes of the ${ }^{13} \mathrm{CO}(3-2)$ and $\mathrm{C}^{18} \mathrm{O}(3-2)$ emitting regions must be $>16^{\prime \prime}$ $(>0.4 \mathrm{pc})$. However, converting the derived mass to a ${ }^{13} \mathrm{CO}$ column density, we predict that the individual condensations have sufficient column densities $\left(\sim 10^{23} \mathrm{~cm}^{-2}\right)$ to be detected in ${ }^{13} \mathrm{CO}(3-2)$ emission in the current observations (peak $\mathrm{T} \sim 10 \mathrm{~K}$ and $\Delta \mathrm{V}=5 \mathrm{~km} \mathrm{~s}^{-1}$ ). It might be, therefore, that the ${ }^{13} \mathrm{CO}$ is depleted toward these condensation. It appears that G028.53-00.25 MM1 does not contain any volume of heated gas and likely is a massive, 
cold core. If a protostar does exist within G028.53-00.25 MM1 then it has not yet heated its surrounding gas. Moreover, if it does contain a circumstellar disk, then it would be much bigger and colder compared to the disk surrounding G034.43+00.24 MM1.

Given the core's mass is $\sim 1000 \mathrm{M}_{\odot}$, and assuming a star-formation efficiency of $30 \%$, we estimate that this core should give rise to a cluster of $300 \mathrm{M}_{\odot}$. Using the expression $m_{\max }$ $=1.2 M_{\text {cluster }}^{0.45}$ Larson 2003), which relates the maximum stellar mass in a cluster $\left(m_{\max }\right)$ to the mass of the cluster $\left(M_{\text {cluster }}\right)$, we estimate $m_{\max }$ to be $\sim 16 \mathrm{M}_{\odot}$. Thus, given its mass and a typical initial mass function (IMF), we would expect this core to give rise to $\sim 4$ high-mass $\left(>8 \mathrm{M}_{\odot}\right)$ stars. Because it contains compact condensations, some evidence for star-formation activity but no obvious heating, we suggest that G028.53-00.25 MM1 may be an example of a very early stage in high-mass star-formation. The measured bolometric luminosity $\left(\sim 10^{2} \mathrm{~L}_{\odot}\right)$ may arise from the accretion of material onto the condensations. Given that the most massive condensation may further fragment it is possible that these cold condensations will continue to accrete material and give rise to a cluster of several high-mass protostars.

It is interesting to compare our observations with recent models that suggest that the fragmentation of a core can be suppressed by the increase in its temperature due to accretion onto lower-mass protostars (Krumholz 2006; Krumholz \& McKee 2008). In this scenario, a core's initial temperature and Jeans mass predicts it will fragment into many low-mass condensations. However, as these condensations form and begin to accrete, the temperature increases which, in turn, increases the overall Jeans mass of the core. An increase in temperature to $\sim 100 \mathrm{~K}$ is thought to be sufficient to halt the fragmentation process. To form high-mass cores and avoid further fragmentation, Krumholz \& McKee (2008) find that the cloud's critical column density, $\Sigma$, should be $\gtrsim 1 \mathrm{~g} \mathrm{~cm}^{-2}$ and its luminosity-to-mass ratio, $\mathrm{L} / \mathrm{M}, \gtrsim 10 \mathrm{~L}_{\odot} / \mathrm{M}_{\odot}$. For the core G028.53-00.25 MM1 we find that $\Sigma$ is $\sim 0.4 \mathrm{~g} \mathrm{~cm}^{-2}$ and $\mathrm{L} / \mathrm{M}$ is $\sim 0.3 \mathrm{~L}_{\odot} / \mathrm{M}_{\odot}$ (Table1). These rough estimates are smaller than the predicted thresholds. Thus, fragmentation and the formation of lower-mass protostars is still possible within G028.53-00.25 MM1. This is in contrast to G034.43+00.24 MM1 which has a measured $\Sigma$ and $\mathrm{L} / \mathrm{M}$ much greater than these thresholds implying that it will give rise to a high-mass star. In this case, however, its high $\mathrm{M}_{G}$ to $\mathrm{M}_{J}$ ratio (for $\mathrm{T}_{D}=100 \mathrm{~K}$ ) suggests that the increase in gas temperature alone may not be enough to halt thermal fragmentation.

The ongoing fragmentation within G028.53-00.25 MM1 is consistent with our detection of multiple condensations toward its center. If this model is correct, then it appears that only the condensation A has sufficient column density to form a high-mass star, the other condensations will most likely only form low-mass stars (Table 3). The derived temperature for the dust within this core is well below $100 \mathrm{~K}$, suggesting that material has not been heated sufficiently to halt the fragmentation. Due to its high mass, this represents an excellent core 
in which to test these theoretical predictions for high-mass star-formation.

\section{Conclusions}

Using the SMA we have obtained high-angular resolution sub-millimeter continuum images and molecular line spectra toward two cores within IRDCs. In the low-angular resolution data these cores have sizes $\mathrm{R}<0.8 \mathrm{pc}$, gas masses $\mathrm{M}>400 \mathrm{M}_{\odot}$, and bolometric luminosities $\mathrm{L} \gtrsim 10^{2} \mathrm{~L}_{\odot}$ (Rathborne et al. 2006, 2008). Despite their similar sizes, masses, and bolometric luminosities the two cores show remarkable differences in the high-angular resolution data. The sub-millimeter continuum images reveal that G034.43+00.24 MM1 remains unresolved and has a size of $\sim 0.03 \mathrm{pc}$ and mass of $29 \mathrm{M}_{\odot}$. In contrast, G028.53-00.25 MM1 contains at least three compact $(\sim 0.06 \mathrm{pc})$, massive $\left(9-21 \mathrm{M}_{\odot}\right)$ condensations.

Moreover, the molecular line spectra reveal that G034.43+00.24 MM1 emits strong lines from many complex molecules, but the core G028.53-00.25 MM1 does not. This suggests that G034.43+00.24 MM1 is hot, compact, and dense, while G028.53-00.25 MM1 is colder and possibly more extended. We speculate, therefore, that G034.43+00.24 MM1 is a HMC in an early stage in the formation of a high-mass protostar, while G028.53-00.25 MM1 may represent an even earlier phase in the high-mass star-formation process. Because G028.53-00.25 MM1 shows some evidence for active star formation in the low-angular resolution data (e.g., shocked gas, high velocity line profiles, and a high bolometric luminosity), we cannot rule out the possibility that the condensations within this core may have already begun to accrete. However, the low temperatures and absence of bright $24 \mu \mathrm{m}$ point sources or strong molecular line emission from high excitation transitions suggests that there is little localized heating. Because of its size and mass, G028.53-00.25 MM1 is likely to give rise to at least one high-mass star. We speculate that the detected condensations may continue to accrete material from their surroundings to eventually form high-mass stars. Since the condensations account for only $\sim 4 \%$ of the total mass, there is an ample amount of supply of material to accrete.

The properties of these cores support the idea that high-mass star and cluster formation likely occurs in IRDCs. High-angular sub-millimeter continuum images and molecular line spectra toward more cores within IRDCs are needed to unambiguously establish the evolutionary path from a high-mass starless core to a high-mass protostar.

The authors gratefully acknowledge funding support through NASA grant NNG04GGC92G. This work is based in part on observations made with the Spitzer Space Telescope, which is operated by the Jet Propulsion Laboratory, California Institute of Technology under NASA 
contract 1407. Support for this work was provided by NASA through contract 1267945 issued by JPL/Caltech. The JCMT is operated by JAC, Hilo, on behalf of the parent organizations of the Particle Physics and Astronomy Research Council in the UK, the National Research Council in Canada, and the Scientific Research Organization of the Netherlands. IRAM is supported by INSU/CNRS (France), MPG (Germany), and IGN (Spain). We would also like to thank Steven Longmore and Mark Krumholz for useful discussions. The authors would also like to thank the anonymous referee for comments that greatly improved the paper.

Facilities: SMA, IRAM, JCMT, Spitzer.

\section{REFERENCES}

Beuther, H., Schilke, P., Sridharan, T. K., Menten, K. M., Walmsley, C. M., \& Wyrowski, F. 2002, A\&A, 383, 892

Beuther, H., Sridharan, T. K., \& Saito, M. 2005, ApJ, 634, L185

Bonnell, I. A. \& Bate, M. R. 2002, MNRAS, 336, 659

Carey, S. J., Clark, F. O., Egan, M. P., Price, S. D., Shipman, R. F., \& Kuchar, T. A. 1998, ApJ, 508, 721

Carey, S. J., Feldman, P. A., Redman, R. O., Egan, M. P., MacLeod, J. M., \& Price, S. D. 2000, ApJ, 543, L157

Cesaroni, R., Galli, D., Lodato, G., Walmsley, C. M., \& Zhang, Q. 2007, in Protostars and Planets V, ed. B. Reipurth, D. Jewitt, \& K. Keil, 197-212

Chambers, E. T., Jackson, J. M., Rathborne, J. M., \& Simon, R. 2008, ApJ, in preparation

Churchwell, E. 2002, ARA\&A, 40, 27

Egan, M. P., Shipman, R. F., Price, S. D., Carey, S. J., Clark, F. O., \& Cohen, M. 1998, ApJ, 494, L199

Garay, G., Faúndez, S., Mardones, D., Bronfman, L., Chini, R., \& Nyman, L.-Å. 2004, ApJ, 610,313

Garay, G. \& Lizano, S. 1999, PASP, 111, 1049

Hildebrand, R. H. 1983, QJRAS, 24, 267

Ho, P. T. P., Moran, J. M., \& Lo, K. Y. 2004, ApJ, 616, L1 
Krumholz, M. R. 2006, ApJ, 641, L45

Krumholz, M. R. \& McKee, C. F. 2008, ArXiv e-prints, 801

Kurtz, S., Cesaroni, R., Churchwell, E., Hofner, P., \& Walmsley, C. M. 2000, Protostars and Planets IV, 299

Larson, R. B. 2003, in Astronomical Society of the Pacific Conference Series, Vol. 287, Galactic Star Formation Across the Stellar Mass Spectrum, ed. J. M. De Buizer \& N. S. van der Bliek, 65-80

Lis, D. C. \& Carlstrom, J. E. 1994, ApJ, 424, 189

McKee, C. F. \& Tan, J. C. 2003, ApJ, 585, 850

Ormel, C. W., Shipman, R. F., Ossenkopf, V., \& Helmich, F. P. 2005, A\&A, 439, 613

Ossenkopf, V. \& Henning, T. 1994, A\&A, 291, 943

Pillai, T., Wyrowski, F., Menten, K. M., \& Krügel, E. 2006, A\&A, 447, 929

Rathborne, J. M., Jackson, J. M., Chambers, E. T., Simon, R., Shipman, R., \& Frieswijk, W. 2005, ApJ, 630, L181

-. 2008, ApJ, in preparation

Rathborne, J. M., Jackson, J. M., \& Simon, R. 2006, ApJ, 641, 389

Rathborne, J. M., Simon, R., \& Jackson, J. M. 2007, ApJ, 662, 1082

Redman, R. O., Feldman, P. A., Wyrowski, F., Côté, S., Carey, S. J., \& Egan, M. P. 2003, ApJ, 586, 1127

Shepherd, D. S., Nürnberger, D. E. A., \& Bronfman, L. 2004, ApJ, 602, 850

Shepherd, D. S., Povich, M. S., Whitney, B. A., Robitaille, T. P., Nürnberger, D. E. A., Bronfman, L., Stark, D. P., Indebetouw, R., Meade, M. R., \& Babler, B. L. 2007, ApJ, 669, 464

Simon, R., Jackson, J. M., Rathborne, J. M., \& Chambers, E. T. 2006a, ApJ, 639, 227

Simon, R., Rathborne, J. M., Shah, R. Y., Jackson, J. M., \& Chambers, E. T. 2006b, ApJ, 653,1325

Wang, Y., Zhang, Q., Rathborne, J. M., Jackson, J., \& Wu, Y. 2006, ApJ, 651, L125 
Zhang, Q. 2005, in IAU Symposium, Vol. 227, Massive Star Birth: A Crossroads of Astrophysics, ed. R. Cesaroni, M. Felli, E. Churchwell, \& M. Walmsley, 135-144

Zhang, Q., Hunter, T. R., Brand, J., Sridharan, T. K., Cesaroni, R., Molinari, S., Wang, J., \& Kramer, M. 2005, ApJ, 625, 864

Zhang, Q., Hunter, T. R., Brand, J., Sridharan, T. K., Molinari, S., Kramer, M. A., \& Cesaroni, R. 2001, ApJ, 552, L167

Zinnecker, H., \& Yorke, H. W. 2007, ARA\&A, 45, 481 
Table 1: Core properties

\begin{tabular}{|c|c|c|c|c|c|c|c|c|c|c|c|c|c|c|}
\hline \multirow[t]{2}{*}{ Core } & \multicolumn{2}{|c|}{ Coordinates } & \multirow{2}{*}{$\begin{array}{c}\mathrm{V}_{L S R} \\
\left(\mathrm{~km} \mathrm{~s}^{-1}\right)\end{array}$} & \multirow{2}{*}{$\begin{array}{c}\mathrm{D} \\
(\mathrm{kpc}) \\
\end{array}$} & \multirow{2}{*}{$\begin{array}{c}\mathrm{R} \\
(\mathrm{pc}) \\
\end{array}$} & \multirow{2}{*}{$\begin{array}{l}\mathrm{T}_{D} \\
(\mathrm{~K}) \\
\end{array}$} & \multirow[t]{2}{*}{$\beta$} & \multirow{2}{*}{$\begin{array}{c}\mathrm{L} \\
\left(\mathrm{L}_{\odot}\right) \\
\end{array}$} & \multirow{2}{*}{$\begin{array}{c}\mathrm{M}_{G} \\
\left(\mathrm{M}_{\odot}\right) \\
\end{array}$} & \multirow[t]{2}{*}{$\frac{M_{G}}{M_{J}}$} & \multirow{2}{*}{$\bar{~} \frac{M_{G}}{M_{V}}$} & \multirow{2}{*}{$\begin{array}{l}\lambda_{J} \\
(\mathrm{pc}) \\
\end{array}$} & \multirow[t]{2}{*}{$\bar{\Sigma} \Sigma$} & \multirow[t]{2}{*}{ 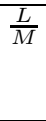 } \\
\hline & $\begin{array}{c}\mathrm{RA} \\
(\mathrm{J} 2000)\end{array}$ & $\begin{array}{c}\text { Dec } \\
(\mathrm{J} 2000)\end{array}$ & & & & & & & & & & & & \\
\hline G034.43+00.24 MM1 & $18: 53: 18.0$ & $01: 25: 24$ & 57 & 3.7 & 0.10 & 34 & 1.5 & 19,600 & 430 & 550 & 2.8 & 0.04 & 3.2 & 46 \\
\hline G028.53-00.25 MM1 & $18: 44: 18.0$ & $-03: 59: 34$ & 87 & 5.7 & 0.42 & 16 & 1.2 & 370 & 1130 & 800 & 2.3 & 0.16 & 0.4 & 0.3 \\
\hline References & & & 1 & 1 & 2 & 3 & 3 & 3 & 3 & 4 & 4 & 4 & 4 & 4 \\
\hline
\end{tabular}

References. - (1) Simon et al. (2006b); (2) Rathborne et al. (2006); (3) Rathborne et al. (2008); (4) this work.

Table 2: Summary of the low-angular resolution molecular line spectra

\begin{tabular}{|c|c|c|c|c|c|c|c|c|}
\hline \multirow[t]{2}{*}{ Molecular line } & \multicolumn{4}{|c|}{ G034.43+00.24 MM1 } & \multicolumn{4}{|c|}{ G028.53-00.25 MM1 } \\
\hline & $\begin{array}{l}\mathrm{T}_{A}^{*} \\
(\mathrm{~K})\end{array}$ & $\begin{array}{c}\mathrm{V}_{L S R} \\
\left(\mathrm{~km} \mathrm{~s}^{-1}\right)\end{array}$ & $\begin{array}{c}\Delta V \\
\left(\mathrm{~km} \mathrm{~s}^{-1}\right)\end{array}$ & $\begin{array}{c}\mathrm{I}^{\mathrm{a}} \\
\left(\mathrm{K} \mathrm{km} \mathrm{s}^{-1}\right)\end{array}$ & $\begin{array}{l}\mathrm{T}_{A}^{*} \\
(\mathrm{~K})\end{array}$ & $\begin{array}{c}\mathrm{V}_{L S R} \\
\left(\mathrm{~km} \mathrm{~s}^{-1}\right)\end{array}$ & $\begin{array}{c}\Delta V \\
\left(\mathrm{~km} \mathrm{~s}^{-1}\right)\end{array}$ & $\begin{array}{c}\mathrm{I}^{\mathrm{a}} \\
\left(\mathrm{K} \mathrm{km} \mathrm{s}^{-1}\right)\end{array}$ \\
\hline $\mathrm{C}^{18} \mathrm{O}(3-2)$ & 2.9 & 57.8 & 3.7 & 11.1 & 1.2 & 87.0 & 3.2 & 4.8 \\
\hline${ }^{13} \mathrm{CO}(3-2)$ & 8.2 & 57.6 & 5.1 & 44.8 & 2.4 & 86.2 & 5.8 & 14.8 \\
\hline $\mathrm{HCN}(4-3)$ & 3.4 & 57.7 & 12.5 & 45.8 & 1.2 & 86.0 & 7.6 & 1.1 \\
\hline CS $(3-2)$ & 4.1 & 57.9 & 6.8 & 31.7 & 0.5 & 86.1 & 7.4 & 4.2 \\
\hline $\mathrm{N}_{2} \mathrm{H}^{+}(1-0)$ & $1.0^{\mathrm{b}}$ & 57.8 & 2.6 & 57.1 & $0.2^{\mathrm{b}}$ & 86.8 & 3.6 & 16.4 \\
\hline $\mathrm{SiO}(2-1)$ & 0.8 & 57.9 & 10.6 & 9.3 & 0.3 & 86.4 & 9.0 & 3.2 \\
\hline $\mathrm{H}^{13} \mathrm{CO}^{+}(1-0)$ & 0.8 & 57.9 & 10.5 & 9.0 & 0.3 & 86.4 & 9.1 & 3.3 \\
\hline $\mathrm{HCO}^{+}(1-0)$ & 2.5 & 60.2 & 3.7 & 13.4 & 1.1 & 87.5 & 9.2 & 11.2 \\
\hline
\end{tabular}

${ }^{a}$ The integrated intensity quoted here is not the area under the Gaussian, but instead is calculated over a velocity range to include all the emission.

${ }^{b}$ This is $\tau \mathrm{T}_{A}^{*}$ for the main hyperfine components.

Table 3: Condensation properties

\begin{tabular}{|c|c|c|c|c|c|c|c|c|c|c|c|}
\hline \multirow[t]{2}{*}{ Condensation } & \multicolumn{2}{|c|}{ Coordinates } & \multirow{2}{*}{$\begin{array}{c}\text { Peak } \\
\text { flux } \\
(\mathrm{mJy}) \\
\end{array}$} & \multirow{2}{*}{$\begin{array}{c}\text { Deconvoled } \\
\text { diameter } \\
(\mathrm{pc})\end{array}$} & \multirow{2}{*}{$\begin{array}{l}\mathrm{T}_{D} \\
(\mathrm{~K}) \\
\end{array}$} & \multirow[t]{2}{*}{$\beta$} & \multirow{2}{*}{$\begin{array}{c}\mathrm{M}_{G} \\
\left(\mathrm{M}_{\odot}\right) \\
\end{array}$} & \multirow{2}{*}{$\frac{M_{G}}{M_{J}}$} & \multirow{2}{*}{$\frac{M_{G}}{M_{V}}$} & \multirow{2}{*}{$\begin{array}{l}\lambda_{J} \\
(\mathrm{pc}) \\
\end{array}$} & \multirow[t]{2}{*}{$\bar{\Sigma}$} \\
\hline & $\begin{array}{c}\mathrm{RA} \\
(\mathrm{J} 2000) \\
\end{array}$ & $\begin{array}{c}\text { Dec } \\
(\mathrm{J} 2000) \\
\end{array}$ & & & & & & & & & \\
\hline G034.43+00.24 MM1 & $18: 53: 18.03$ & $1: 25: 25.58$ & 2760 & 0.03 & 100 & 1.5 & 29 & 30 & 1.2 & 0.02 & 7.7 \\
\hline G028.53-00.25 MM1 A & $18: 44: 18.08$ & $-3: 59: 34.33$ & 73 & 0.05 & 16 & 1.2 & 21 & 160 & 0.8 & 0.01 & 2.7 \\
\hline G028.53-00.25 MM1 B & 18:44:17.95 & $-3: 59: 31.27$ & 33 & 0.10 & 16 & 1.2 & 9 & 10 & 0.2 & 0.07 & 0.2 \\
\hline G028.53-00.25 MM1 C & $18: 44: 17.93$ & $-3: 59: 35.42$ & 31 & 0.06 & 16 & 1.2 & 9 & 30 & 0.3 & 0.03 & 0.8 \\
\hline
\end{tabular}



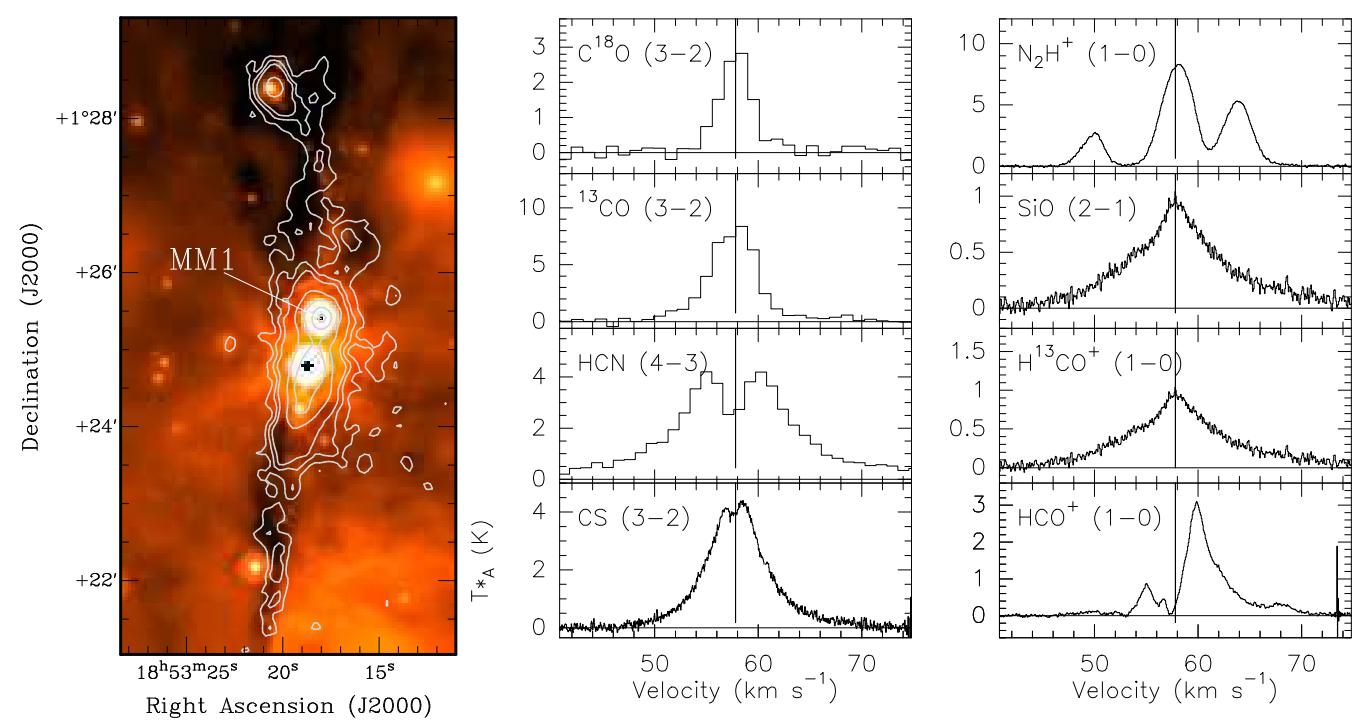

Fig. 1.- G034.43+00.24. Left: Spitzer $24 \mu \mathrm{m}$ image with contours of the IRAM $30 \mathrm{~m}$ $1.2 \mathrm{~mm}$ continuum emission (Rathborne et al. 2006; contour levels are 60, 90, 120, 240, 480, 1200 , and $2200 \mathrm{mJy}$ beam $^{-1}$; color scale is logarithmic from $30 \mathrm{MJy} \mathrm{sr}^{-1}$ [black] to $200 \mathrm{MJy}$ $\mathrm{sr}^{-1}$ [white]). Note that while the IRDC remains dark at $24 \mu \mathrm{m}$ the core MM1 is associated with bright $24 \mu \mathrm{m}$ emission. Right: IRAM and JCMT molecular line emission toward the core MM1. The solid vertical line marks the central velocity of the core, as traced by the optically thin $\mathrm{C}^{18} \mathrm{O}(3-2)$ emission. Note the broad line widths in the high-density tracers, CS (3-2) and HCN (4-3), the shocked gas as traced by the bright $\mathrm{SiO}(2-1)$ emission and the high velocity line wings in $\mathrm{HCO}^{+}(1-0)$ and $\mathrm{H}^{13} \mathrm{CO}^{+}(1-0)$. Table 2 summarizes the results of fits to these spectra. 

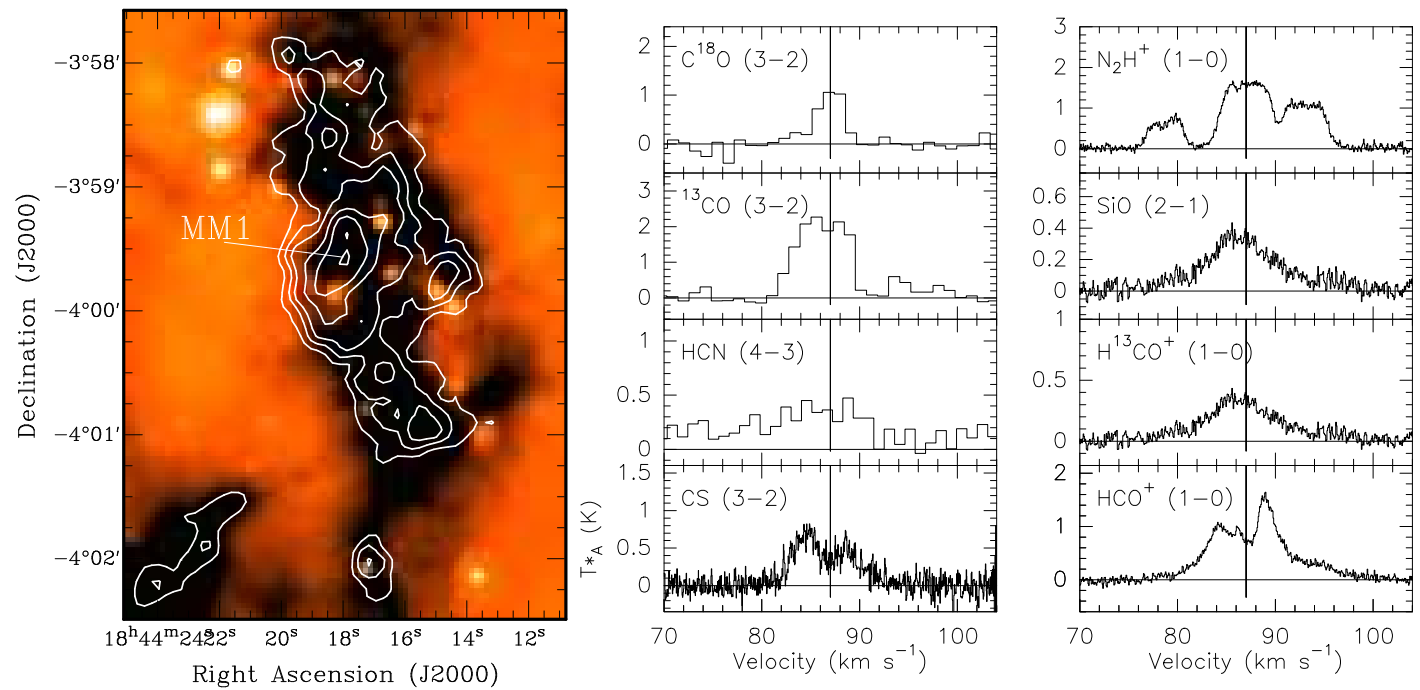

Fig. 2.- G028.53-00.25. Left: Spitzer $24 \mu \mathrm{m}$ image with contours of the IRAM $30 \mathrm{~m}$ $1.2 \mathrm{~mm}$ continuum emission (Rathborne et al. 2006; contour levels are 60, 90, 120, 180, 240, and $320 \mathrm{mJy}_{\text {beam }}{ }^{-1}$; color scale is logarithmic with $50 \mathrm{MJy} \mathrm{sr}^{-1}$ [black] to $100 \mathrm{MJy} \mathrm{sr}^{-1}$ [white]). Note that the IRDC and, in particular, the core MM1 remain dark at $24 \mu \mathrm{m}$. Right: IRAM and JCMT molecular line emission toward the core MM1. The solid vertical line marks the central velocity of the core, as traced by the optically thin $\mathrm{C}^{18} \mathrm{O}(3-2)$ emission. Note the saturated ${ }^{13} \mathrm{CO}(3-2)$ and $\mathrm{N}_{2} \mathrm{H}^{+}(1-0)$ line emission and the faint emission from $\mathrm{CS}(3-2)$ and $\mathrm{HCN}(4-3)$. The presence of broad line wings in the $\mathrm{SiO}(2-1), \mathrm{HCO}^{+}(1-0)$, and $\mathrm{H}^{13} \mathrm{CO}^{+}(1-0)$, spectra suggests that some star-formation activity is occurring within this core. Table 2 summarizes the results of fits to these spectra. 

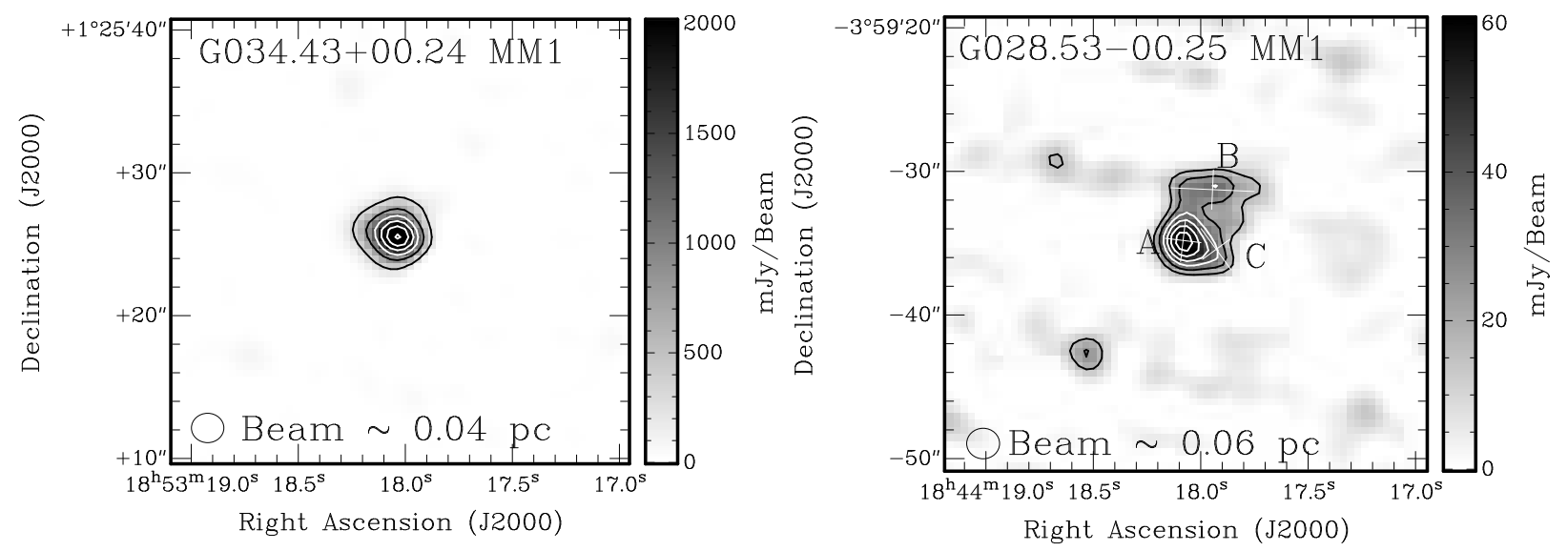

Fig. 3.- SMA continuum images toward the two cores (the images cover $\sim 30^{\prime \prime}$ around the core's center). The contour levels for G034.43+00.24 MM1 (left) are 0.3 to 3.0 in steps of $0.5 \mathrm{Jy} \mathrm{beam}^{-1}$ and for G028.53-00.25 MM1 (right) are 0.015 to 0.07 in steps of $0.01 \mathrm{Jy} \mathrm{beam}^{-1}$. In both cases the lowest two contour levels are in black for clarity. Note that G034.43+00.24 MM1 contains a single, compact ( $\sim 0.03 \mathrm{pc})$, massive $\left(24 \mathrm{M}_{\odot}\right)$ emission feature, while G028.53-00.25 MM1 appears resolved into three individual condensations. These condensations are marked with a cross and have sizes of $\sim 0.06 \mathrm{pc}$ and masses of 8-19 $\mathrm{M}_{\odot}$. Table 3 list their properties. 


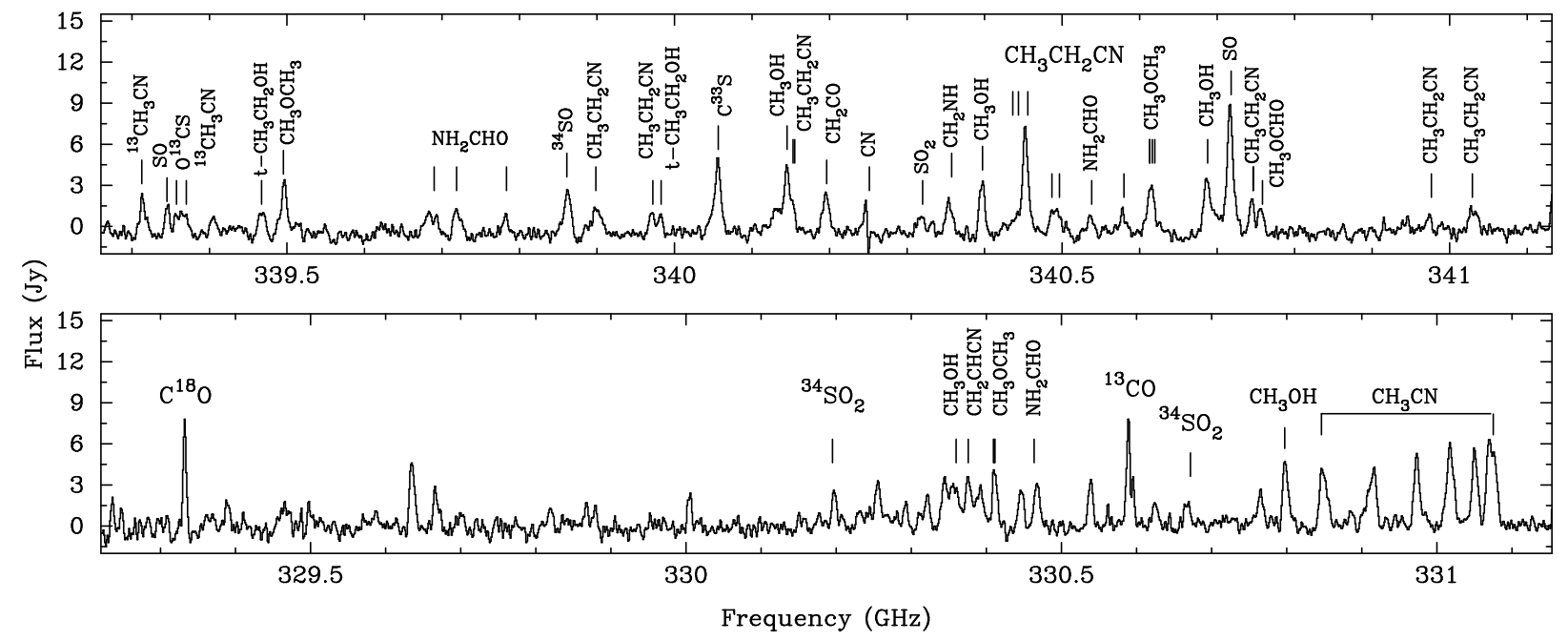

Fig. 4. - SMA spectra toward G034.43+00.24 MM1. Both the lower and upper sidebands are shown (upper and lower panels respectively). The spectra are averaged over a $\sim 2^{\prime \prime}$ region centered on the peak in the high-angular resolution continuum image. Note the strong emission from the many complex molecules. Thus, we suggest that G034.43+00.24 MM1 is a HMC associated with the very earliest stage in the formation of a high-mass protostar. The molecular lines marked on this figure were based on the output from the Lovas on-line catalog of suggested rest frequencies. 


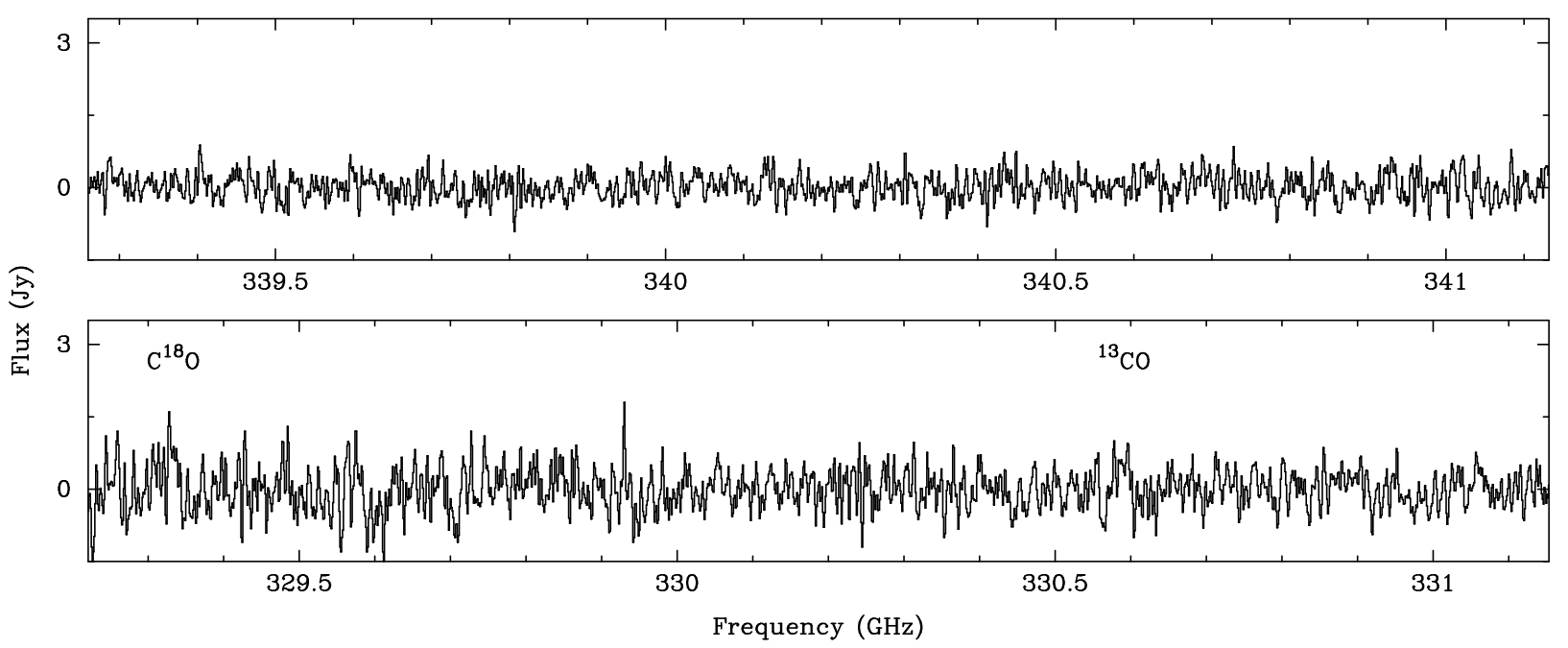

Fig. 5.- SMA spectra toward G028.53-00.25 MM1. Both the lower and upper sidebands are shown (upper and lower panels respectively). The spectra are averaged over a $\sim 2^{\prime \prime}$ region centered on the peak in the high-angular resolution continuum image. While ${ }^{13} \mathrm{CO}(3-2)$ and $\mathrm{C}^{18} \mathrm{O}(3-2)$ emission is clearly seen toward this core in the lower-angular resolution data (Fig. 2, 14" beam), very little is seen in this spectrum. The absence of strong ${ }^{13} \mathrm{CO}(3-2)$ or $\mathrm{C}^{18} \mathrm{O}(3-2)$ emission in the high-angular resolution spectrum may imply that the emission seen in the lower-angular resolution spectrum arises from a cold extended envelope which is resolved out by the interferometer.

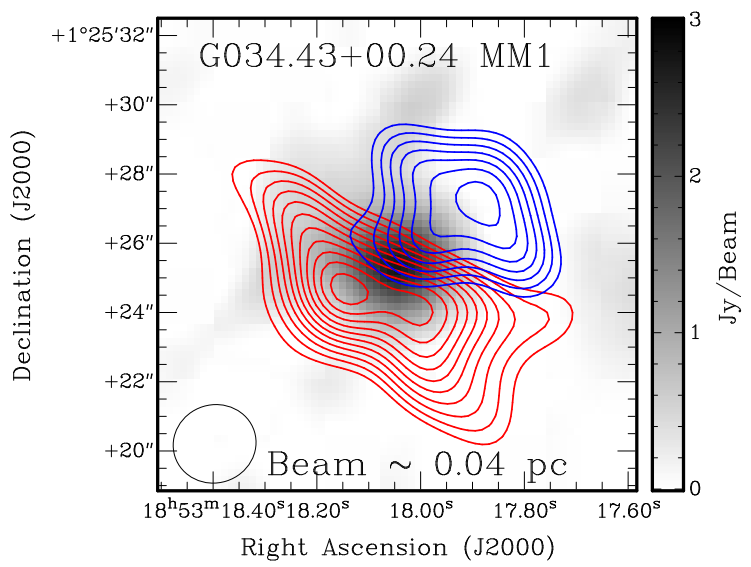

Fig. 6.- SMA $\mathrm{CH}_{3} \mathrm{CH}_{2} \mathrm{CN}$ integrated intensity image in gray scale tracing the unresolved HMC within G034.43+00.24 MM1. Contours show the red-shifted $\left(\mathrm{V}_{L S R}=61 \mathrm{~km} \mathrm{~s}^{-1}\right)$ and blue-shifted $\left(\mathrm{V}_{L S R}=55 \mathrm{~km} \mathrm{~s}^{-1}\right){ }^{13} \mathrm{CO}(3-2)$ emission surrounding the unresolved, bright HMC (emission is from a single channel, with the same contour levels for the red and blue components; 1.7 to 7.5 in steps of $0.5 \mathrm{Jy}_{\text {beam }}^{-1}$ ). This emission appears to be tracing an extended rotating envelope. 


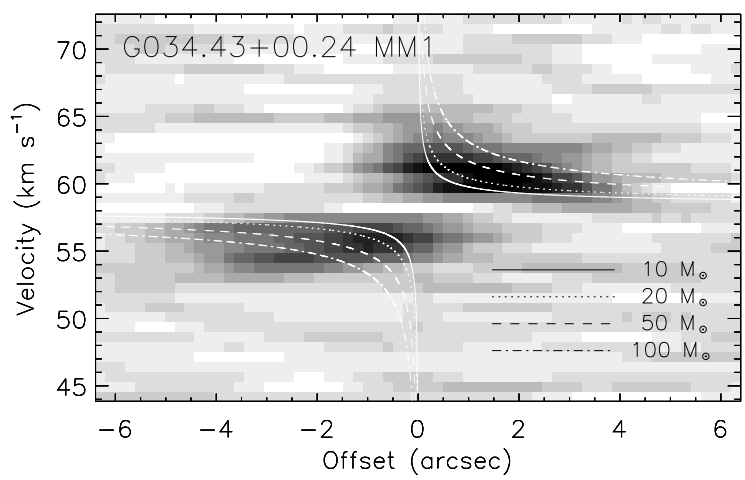

Fig. 7.- SMA ${ }^{13} \mathrm{CO}(3-2)$ position-velocity diagram for G034.43+00.24 MM1. The slice is taken perpendicular to the axis of the rotating envelope and shows the two components. Overlaid on this image are models for Keplerian rotation with central masses of 10, 20, 50, and $100 \mathrm{M}_{\odot}$. The emission from G034.43+00.24 MM1 is consistent with Keplerian rotation around $\mathrm{a} \sim 10-50 \mathrm{M}_{\odot}$ source. 